\title{
GEOLOGÍA DE LA HOJA TIERRAS MORENAS
}

\author{
Giovanna Civelli ${ }^{1}$, Umberto Locati $^{1}$, Biagio Bigioggero ${ }^{2}$, Sergio Chiesa $^{2}$, \\ Guillermo E. Alvarado ${ }^{3 *}$ y Oscar Mora ${ }^{3}$ \\ ${ }^{1}$ ERA Soc. Coop. Via Pasubio 3/5, 24044 Dalmine (Bergamo), Italia \\ ${ }^{2}$ CNR-IDPA, Via Pasubio 3/5, 24044 Dalmine (Bergamo), Italia \\ ${ }^{3}$ Instituto Costarricense de Electricidad, Apdo. 10032-1000, Costa Rica \\ *Autor para contacto: galvaradoi@ice.go.cr
}

(Recibido 15/06/06; aceptado 03/11/06)

\section{INTRODUCCIÓN}

Los primeros mapas geológicos que cubren parte de la hoja Tierras Morenas, se remontan a los estudios de reconocimiento realizados por César Dondoli, Gabriel Dengo, Rodolfo Madrigal, y Alfredo Mainieri, entre otros. Sin embargo, tan solo hasta hace unos pocos años se realizó una cartografía y estratigrafía de detalle de los depósitos volcánicos y epiclásticos que afloran en el área como parte de un convenio entre el Instituto Costarricense de Electricidad (ICE) el Consejo Nacional de Investicaciones (CNR) y la Universidad de Milán, Italia. Un grupo de estudiantes italianos llegaron a Costa Rica para realizar el mapeo de la hoja Tierras Morenas (entre otras que le sucedieron) como parte de su proyecto de tesis e investigación a finales de los 80s (Chiesa et al., 1987; Mora, 1988; Civelli, 1990; Locati, 1990, Pecchio, 1990). Los resultados de dicho mapeo, fueron presentados en la Revista Geológica de América Central en

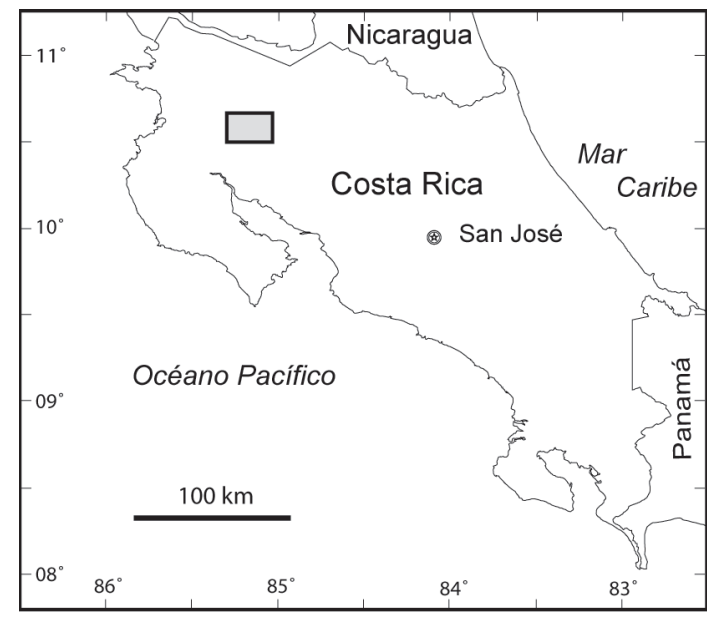

Fig. 1: Localización de la hoja Tierras Morenas

1992 (Chiesa et al., 1992). Sin embargo, un mapa a una escala más adecuada nunca fue publicado, por lo que ahora se presenta con modificaciones y actualizaciones con respecto al original, y a la luz de nuevos datos estratigráficos y cronológicos, 
dada su importancia por estar en una región de gran desarrollo desde diversos puntos de vista (agricultura, irrigación, ganadería, infraestructura, generación eléctrica, geoturismo, arqueológica). El sector estudiado se ubica al sur del los volcanes Miravalles y Tenorio, y está cubierto por unidades de flujos piroclásticos intercalados con depósitos de caída, sedimentos continentales, paleosuelos bien desarrollados y lavas.

A continuación, una síntesis de las unidades estratigráficas y la estructura geológica. Se usan los nombres estratigráficos tradicionales (verbigracia, formaciones Bagaces y Monteverde), pero para las unidades de menor rango dentro de ellos, se usan nominaciones informales, en tanto que aún es imprescindible un trabajo de definición regional de los detalles estratigráficos de tales unidades volcánicas. El presente trabajo es una continuidad geográfica y geológica del mapa geológico de la hoja Cañas, ubicada directamente al sur (Aiazzi et al., 2004).

\section{ESTRATIGRAFÍA}

\section{Flujos soldados (Formación Bagaces)}

Descripción: Está constituido por ignimbritas soldadas con fiammes, intercaladas localmente con depósitos epiclásticos. Suelen ser negras, densas y vítreas con fenocristales de plagioclasa. Pueden adquirir una estructura prismática característica. Su espesor es de al menos $40 \mathrm{~m}$.

Localidades: Buenos afloramientos se observan a lo largo de la Carretera Interamericana, en el sector suroeste del mapa.

Aspectos regionales: Afloran a lo largo de toda la vertiente pacífica de la cordillera de Guanacaste (meseta de Santa Rosa).

Relaciones estratigráficas: Sobreyace localmente a las lavas de Taboga (Grupo Aguacate), ubicadas mas al SE (ver Aiazzi et al., 2004).

Edad: No hay edades precisas de las ignimbritas en esta zona, aunque por correlación (hoja
Cañas, Aiazzi et al., 2004) se asume una edad Pliocena Inferior.

Aspectos específicos: Se requieren de más estudios estratigráficos, vulcanológicos y geoquímicos. Algunos trabajos ya están en proceso, para ser publicados a corto plazo.

Antecedentes: Una serie de informes inéditos del ICE, así como los trabajos regionales de Dengo (1962), Chiesa et al. $(1987,1992)$ y Vogel et al. (2004).

\section{Flujos pumíticos no soldados (Formación Bagaces)}

Descripción: Está constituido por varias unidades de flujos de pómez, caracterizados por diferentes contenidos en pómez y cristales de cuarzo, plagioclasa, anfiboles y rara biotita. Cada flujo tiene un área de afloramientos diferente, generalmente bien distinguible y está intercalado a los otros flujos con paleosuelos y oleadas piroclásticas. Su espesor alcanza $>10 \mathrm{~m}$. Los flujos de Montano comprenden por lo menos cuatro diferentes flujos de pómez separados por paleosuelos, depósitos de caída y niveles volcanoclásticos retrabajados. Cada unidad de flujo está constituida por pómez (5-50\%), fragmentos líticos (10-20\%) y matriz de ceniza (50-90\%). Las pómez tienen color claro y dimensiones comprendidas entre 2 y $8 \mathrm{~cm}$. Los líticos (5-15 $\%$ ) son casi siempre pequeños (aprox. $1 \mathrm{~mm}$ ) aunque pueden alcanzar unos $2 \mathrm{~cm}$ y están constituidos por lavas. La característica común de todas las unidades es la casi total falta de cristales $(<3$ $\%)$ en las pómez y en la matriz.

Localidades: Afloramientos se observan en el sector centro-occidental del área de estudio, entre el río Tenorio hacia el este y La Ese hacia el norte. En particular hay buenos afloramientos cerca del Sitio Cacao, hacia el Río Aguacaliente y cerca de las Cuestas Cambalache y Jobo. Referente a los flujos de Montano, una buena localidad se ubica cerca del pueblo de Montano $(405 \mathrm{~N}-280 \mathrm{E})$. 
Aspectos regionales: Los flujos Montano afloran en un área de $30 \mathrm{~km}^{2}$ y representan sin duda una facies distal (escaso espesor, abundancia de matriz de cenizas, líticos pequeños), por el momento de procedencia desconocida. Sin embargo, presentan algunas afinidades con otro flujo, aflorante al este del río Tenorio hasta la ciudad de Cañas, denominado preliminarmente como flujo de Sandillal. Por eso, se ha preferido incluirlos, por el momento, en esta formación y atribuirlos a una procedencia más oriental (área el volcán Tenorio). Su espesor medio es de unos 20 m (Mora, 1988; Civelli, 1990).

Relaciones estratigráficas: Sobreyacen localmente a las lavas del Grupo Aguacate y están subyacidos por depósitos epiclásticos y por las lavas de Monteverde. Chiesa et al. (1992) hablan de unidades como Formación Sandillal y los flujos de Montano, pero a la luz de los datos geoquímicos y cronoestratigráficos (p.ej. Vogel et al., 2004), se requieren de estudios complementarios para una mejor redefinición.

Edad geológica: Las edades ${ }^{40} \mathrm{Ar} /{ }^{39} \mathrm{Ar}$ suministran edades para los eventos pumíticos aflorantes en el área, de 4,15; 2,06 y 2 Ma (Vogel et al, 2004), correspondientes al Plioceno.

Aspectos específicos: Se requieren de más estudios para dilucidar su procedencia con base en detalles estratigráficos, vulcanológicos y geoquímicos. Algunos trabajos ya están en proceso a ser publicados a corto plazo.

Antecedentes: Chiesa et al. (1992) describen algunos flujos piroclásticos aflorantes en el área. Vogel et al. (2004) realizan un estudio petrológico con cierto grado de detalle e incluyen algunas dataciones ${ }^{40} \mathrm{Ar} /{ }^{39} \mathrm{Ar}$.

\section{Lavas del cerro Las Nubes (Formación Monteverde)}

Descripción: Está constituida por coladas andesíticas, con diferentes niveles en correspondencia con diversos frentes de coladas.
Localidades: Afloran en el sector oriental del mapa, al este del río Corobicí en una amplia plataforma al sur de Palmira. Hacia el norte están en contacto con el lahar del Tenorio y constituyen un marcado escarpe morfológico.

Aspectos regionales: Constituye la prolongación más occidental de la Formación Monteverde. Otros relieves coetáneos más al $\mathrm{NE}$, poseen morfologías más empinadas y no de meseta, producto de estar más cerca de su fuente y por estar afectadas por fallamiento arqueado.

Relaciones estratigráficas: Claramente sobreyace a tobas pumíticas y sedimentos epiclásticos de la Formación Bagaces y a lavas hidrotermalizadas del Aguacate.

Edad geológica: Las dataciones radiométricas en el área de estudio indican edades con ${ }^{40} \mathrm{Ar} /{ }^{39} \mathrm{Ar}$ de 1,77-1,70 Ma (Pleistoceno Inferior; Gardner \& Turrin en Alvarado et al, 1992). Acorde con las edades regionales, el vulcanismo efusivo de Monteverde se ubica principalmente entre 2 y 1 Ma (Alvarado et al, 1992; Gillot et al., 1994).

Aspectos específicos: Se le reconoce en algunos sectores por su típica forma de mesetas de lava y por lavas frescas, mientras que en otros sectores, no resulta tan obvia la distinción.

Antecedentes: Trabajos anteriores que las mencionan incluyen a Ramírez (1973), quien denomina a dichas lavas como Tierras Morenas, Chaves \& Saénz (1974) y Cigolini \& Chaves (1986) la describen y denominan formalmente corno Formación Monteverde y Mora (1977) como lavas Las Pulgas. Para algunos autores, Monteverde forma parte del Aguacate (cf. Chaves \& Sáenz, 1974), mientras que para otros debe separarse dado que forma parte de un vulcanismo del Cuaternario, y se reconoce por la presencia de plataformas andesíticas y basálticas con edades entre 1 y 2 Ma (Alvarado et al, 1992; Gillot et al., 1994). 


\section{Flujo de pómez del río Liberia}

Descripción: Es un flujo pumítico constituído por abundante matriz (50-70\%), pómez blancas y redondeadas (10-30\%), fragmentos líticos (10-20\%) y cristales de cuarzo, biotita, plagioclasa y pocos anfíboles (15-30\% en total).

Localidades: Una localidad muy conocida por los vulcanólogos es la llamada $\mathrm{km} \mathrm{4}$, con respecto a Bagaces, camino a Guayabo. Por otro lado, cerca de Guayabo aflora otro flujo con biotita similar, pero geoquímicamente diferente.

Aspectos regionales: $\mathrm{Su}$ área de extensión original corresponde a $3500-4000 \mathrm{~km}^{2}$, aproximadamente; por lo tanto, esta unidad representa un excelente estrato guía (Chiesa, 1991).

Relaciones estratigráficas: Sobreyace a los flujos Pliocénicos de la Formación Bagaces (flujos de Montano, Sandillal, Cañas, etc.), e infrayace a los flujos Salitral, Buena Vista y La Ese, entre otros.

Edad geológica: Se le ha datado por K-Ar y ${ }^{40} \mathrm{Ar} / 39 \mathrm{Ar}$ en $1,45-1,9$ Ma (Alvarado et al., 1992, Gillot et al., 1994; Vogel et al., 2004).

Aspectos específicos: Fácilmente reconocible por su abundancia de biotita como fenocristal, seguido de cuarzo y a veces anfíbol. Se toma como la base y capa guía del Pleistoceno Inferior.

Antecedentes: Dengo (1962) define la Formación Liberia como "una toba blanca..., en muchas partes en forma poco coherente..., aparece formada por pequeños fragmentos de pómez y granos irregulares de cuarzo, oligoclasa, sanidina, biotita y magnetita encerrados en una matriz vítrea aglutinada" características que, en su mayor parte, se refieren al flujo de pómez biotítica del río Liberia (véase Chiesa,1991).

\section{Flujo de pómez de "Guayabo"}

Descripción: Los flujos han sido separadas en diferentes unidades con base en su textura, posición estratigráfica, color, contenidos de cristales-pómez-líticos y mineralogía. La sucesión estratigráfica es la siguiente:

El flujo Salitral está constituido por un flujo que contiene pómez (5-25\%), líticos (5-25 $\%)$ y cristales de plagioclasa (10-15\%), cuarzo, ortopiroxeno y poco anfibol (en suma $10 \%$ ) en una matriz de cenizas gruesas (50-80\%). Las pómez tienen una dimensión media de 2-3 $\mathrm{cm}$, pero pueden alcanzar hasta $30 \mathrm{~cm}$ con una evidente gradación normal desde la base hasta el techo del depósito; generalmente son muy vesiculares y con estructura fluidal. Los líticos tienen siempre naturaleza lávica, a veces escoriácea, color negro a rojizo, forma angulosa y dimensiones de hasta $10 \mathrm{~cm}$. La matriz de ceniza muestra variaciones entre el blanco, el gris y el rosado. Su base está caracterizada por la presencia de un nivel de ceniza y un depósito de caída (lapilli lítico y pumítico, así como ceniza de cristales) que llega hasta $20 \mathrm{~cm}$ de espesor. Internamente se pueden observar en algunos de los afloramientos (p.ej. alrededores de Salitral) niveles de cenizas y niveles ricos en pómez gris, que indican la presencia de diferentes subunidades en el mismo flujo.

El flujo de Buena Vista está constituido por una matriz de ceniza gris claro (40-60\%) con pómez (15-60\%), líticos (5-15\%) y abundantes cristales de anfíbol (15\%), plagioclasa, cuarzo, clinopiroxenos y minerales opacos contenidos en las pómez y en la matriz. Las pómez, bien vesiculares, tienen una dimensi6n media de $3-4 \mathrm{~cm}$, sin embargo, en función de una frecuente gradación desde normal hasta inversa, pueden llegar a los $35 \mathrm{~cm}$. Los líticos alcanzan hasta los $10 \mathrm{~cm}$; tienen composición lávica y están distribuidos de modo homogéneo. En algunos afloramientos (p.ej., en los alrededores de Salitral, unos $2 \mathrm{~km}$ al sur del Torno) se encontraron dentro del flujo niveles de cenizas con laminaciones que indican seguramente la depositación de por lo menos dos unidades de flujo. En la base del flujo Buena 
Vista se observan cuatro diferentes depósitos de caída separados por un paleosuelo y constituidos por cristales de plagioclasa y anfíbol $(50 \%)$ y por pómez (50\%); estos depósitos alcanzan hasta $2 \mathrm{~m}$ de espesor.

Los flujos de La Ese representan las unidades más recientes con por lo menos 3 diferentes flujos piroclásticos constituidos por pómez (60-70\%) y líticos (5-10\%) con escasa matriz de ceniza. Los cristales en las pómez o en la matriz parecen totalmente ausentes (afírica). Las pómez son blancas, redondeadas y llegan hasta $15 \mathrm{~cm}$, pero usualmente son inferiores a 3 $\mathrm{cm}$ de diámetro y con moderada vesicularidad. Los líticos son mucho más reducidos (0,5-1 $\mathrm{cm})$ y tienen forma angulosa y naturaleza lávica. Los diferentes flujos están separados por paleosuelos y niveles retrabajados; frecuentes son también los horizontes oxidados y delgados niveles de cenizas, así como chimeneas de desgasificaci6n Varios niveles de típicas brechas co-ignimbríticas que contienen autolitos microgranodioríticos están presentes. Algunos depósitos lacustres finamente estratificados (cenizas, arenas volcánicas y pumitas sin cristales) con un espesor aflorante de 1,6 $\mathrm{m}$ forman parte de

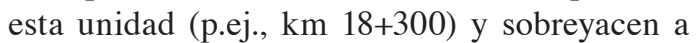
un nivel de pómez plinianas de caída. Sobre el lacustre yacen sedimentos fluviales pumíticos con estratificaci6n inclinada, evidencia de abanicos aluviales que desembocan en pequeñas cuencas lagunares. Más arriba se observan oleadas piroclásticas con lapilli acrecional, y nuevos flujos de oleadas y finalmente un flujo blanco rico en pómez (aprox. $75 \%$ ) con raros anfíboles y líticos (5-10\%) en una matriz ligeramente gris-amarillenta (10-20\%).

Localidades: Una buena localidad para el flujo Salitral es en la Cuesta Lobo (406N284.30E) y para el flujo Buena Vista es la Cuesta Cambalache (407N-280.70E). El flujo La Ese aflora cerca de la antigua escuela del mismo nombre y en antiguos tajos (295.25N-400.60E). En general la transecta entre Bagaces y Guayabo constituye una excelente sección vulcanológica de flujos piroclásticos, quizás una de las mejores y de más fácil acceso del país.
Aspectos regionales: El flujo Salitral es el miembro más extendido de Guayabo, llegando hasta los $200 \mathrm{~km}^{2}$ con un espesor variable entre pocos metros hasta unos $50 \mathrm{~m}$ cerca del borde meridional, de la caldera (San Isidro de Limonal, río Piedras). El flujo Buena Vista es también muy extenso $\left(150 \mathrm{~km}^{2}\right)$ y cerca del borde de la caldera de Guayabo llega a espesores de $30 \mathrm{~m}$. El flujo La Ese con espesores de hasta $40 \mathrm{~m}$, aflora en un área de unos pocos $\mathrm{km}^{2}$, aunque, a la hora de incluir algunos afloramientos aislados, se observa que su área original ha sido mucho mayor.

Relaciones estratigráficas: Las unidades son subyacidas por el flujo del Río Liberia. En la posición estratigráfica superior se pueden observar solamente depósitos volcanoclásticos (lahar del Tenorio y flujos de avalanchas volcánicas de San Bernardo-La Fortuna), depósitos fluviales y lacustre, así como las tetras de caída del volcán Tenorio.

Edad geológica: Las relaciones estratigráficas definidas con base en el trabajo de campo, fueron afinadas por medio de una serie de dataciones obtenidas con el método K-Ar y ${ }^{40} \mathrm{Ar} /{ }^{39} \mathrm{Ar}$. El flujo Salitral fue datado en 1,36 Ma, el Buena Vista en 1,31-1,4 Ma y la última fase fue la que originó el miembro La Ese y se remonta a 0,6-0,9 Ma atrás (Gillot et al., 1994; Vogel et al., 2004).

Aspectos específicos: Aunque dichas unidades litoestratigráficas piroclásticas fueron agrupadas en los flujos de la Formación Guayabo (Chiesa et al., 1992), por proceder aparentemente de la caldera del mismo nombre, sus edades (con excepción de los flujo de La Ese) son más antiguas que las lavas que conforman el borde de la caldera (fechas ${ }^{40} \mathrm{Ar} /{ }^{39} \mathrm{Ar}$ inéditas), lo cual trae un problema en su origen. Un resultado interesante concierne a la comparación entre las formas de las burbujas de las pómez perteneciente al flujo Salitral con respecto al Buena Vista. En el Miembro Salitral las burbujas están constituídas por cavidades redondeadas; mientras que las burbujas del flujo Buena Vista son mucho más 
estiradas, fibrosas y con una porosidad del 35$40 \%$ del volumen total. Los datos muestran que todos los flujos tienen una composición química entre dacítica y riolítica.

Antecedentes: Chiesa et al. (1987), Mora (1988) y Civelli (1990) mapearon dichos fllujos y los agruparon dentro de la Formación Piroclástica Guayabo (Chiesa et al., 1992). Sin embargo, estudios geocronológicos, geoquímicos y de mapeo de detalle han determinado que se trata de muchas unidades de flujos ignimbríticos ácidos provenientes de diferentes centros de emisión, no necesariamente todos ellos asociados con la caldera de Guayabo.

\section{Lavas y piroclastos basálticos del Corobicí}

Descripción: Cono constituido por estratos con gradación inversa de bombas y lapilli inconso-lidadas hasta lapillitas y aglomerados (soldados), con coladas de lava subordinadas de composición basáltica (Chiesa et al, 1994; Alvarado, 2000). Algunas lavas aisladas afloran en las vecindades.

Localidad: Se encuentra a lo largo del Río Corobicí en el sector oriental del mapa, cerca de la finca Santa Cecilia. Otros pequeños afloramientos de basaltos y andesitas basálticas se encuentran a lo largo de la quebrada Ceiba y entre el río Blanco y la Lomas Tigra.

Aspectos regionales: Constituyen una serie de cuatro afloramientos de lavas básicas (incluyendo el cerro Chopo, fuera del mapa) que están mas o menos alineados en sentido NW-SE y ubicados al frente del eje volcánico y que poseen un carácter toleítico.

Relaciones estratigráficas: No son claras dado que los afloramientos y contactos son pobres, pero con seguridad son del Cuaternario, sobreyaciendo a las ignimbritas de Bagaces y a las lavas de Monteverde.
Edad: Presumiblemente Pleistocena pero no hay dataciones radiométricas directas.

Aspectos especificos: Son lavas toleíticas de arco.

Antecedentes: Locati (1990) y Pecchio (1990) son los primeros que cartografían y hacen análisis químicos y petrográficos. Chiesa et al. (1994) publican aspectos químicos.

\section{Lavas del cerro Espiritu Santo}

Descripción: Constituidas por coladas andesíticas con diferentes textura. Generalmente no se encuentran en afloramientos, sino como bloques métricos en los potreros y sectores escarpados.

Localidad: Se encuentran a lo largo de la vertiente meridional del cerro Espíritu Santo. El único buen afloramiento se encuentra a lo largo de la quebrada La Giganta.

Aspectos regionales: Forman parte del vulcanismo que antecede a las cordilleras volcánicas activas.

Edad: Dataciones radiométricas ${ }^{40} \mathrm{Ar} /{ }^{39} \mathrm{Ar}$ no publicadas por P. Gans arrojan edades de alrededor de un millón de años.

Aspectos específicos: Morfológicamente forman parte de los flancos de una antiguo volcán, en el cual todavía se reconocen los valles colgados y sus flanco austral, debido a la formación de una estructura de colapso en su sector norte. Aunque petrográficamente sus lavas son andesíticas, se le asocia con la caldera de Guayabo, cuyo borde de colapso esta constituido por lavas basálticas.

Antecedentes: Los geólogos del ICE e italianos lo habían reconocido en varios trabajos internos como parte de una estructura volcanotectónica. 


\section{Lavas del volcán Tenorio}

Descripción: Lavas y rocas piroclásticas asociadas al vulcanismo y macizo del volcán Tenorio. En el mapa se incluye una subunidad que se refiere a depósitos piroclásticos que afloran cerca de río Chiquito, que han sido poco estudiados.

Localidad: Flanco sur del volcán Tenorio y los depósitos piroclásticos (tefras) de río Chiquito.

Aspectos regionales: Forma parte de los edificios volcánicos que conforma la cordillera de Guanacaste.

Edad: Pleistoceno Medio y Superior.

Aspectos específicos: Volcán sin actividad eruptiva histórica ni holocena comprobada, aunque faltan estudios de detalle. Su morfología es indicio de que ha tenido actividad no muy antigua, geológicamente hablando (Pleistoceno Superior y quizás Holoceno).

Antecedentes: Volcán poco estudiado. Para algunos detalles, ver Alvarado (2000).

\section{Lahares del Tenorio}

Descripción: Al suroeste del cono principal del volcán Tenorio se encuentran unos depósitos epiclásticos descritos como Lahar del Tenorio, pero que en las facies cercanas, por ejemplo en la hacienda Tenorio, poseen una topografía de hummocky, lo cual indica que se trató de un debris avalanche que probablemente por incorporación de agua, o por la presencia de acuíferos en el flanco colapsado del volcán, se transformó en un debris flow. No se distingue con claridad la caldera de avalancha de donde se originó, probablemente porque fue cubierto por actividad posterior del volcán, a juzgar por el cono bien desarrollado con un patrón de drenaje radial juvenil o poco profundizado (Alvarado et al., 2004).
Localidades: Aflora en sector central de la hoja Tierras Morenas, entre los ríos Tenorio y Corobicí.

Aspectos regionales: Proviene del volcán Tenorio.

Relaciones estratigráficas: Cubre a los flujos pumíticos de Bagaces. En una perforación geotérmica se alcanzó al menos un espesor de $200 \mathrm{~m}$

Edad geológica: No se ha determinado, aunque debe de quedar comprendida en el Pleistoceno Superior.

Aspectos específicos: Se trata de un debris avalanche diluido a debris flow.

Antecedentes: Fue reconocido y mapeado por Locati (1989). Alvarado et al. (2004) complementan su descripción e interpretación en la parte proximal.

\section{Debris avalanche de la Fortuna}

Descripción: Al suroeste del cono principal del Miravalles se encuentran unos depósitos epiclásticos descritos como debris avalanche de la Fortuna (antiguamente como de San Bernardo), que cerca de La Fortuna en las facies cercanas poseen una topografía de hummocky.

Localidades: Aflora en sector septentrional de la hoja Tierras Morenas, entre el borde de la caldera de Guayabo, el cerro Espíritu Santo y la plana de Casa Vieja Norte.

Aspectos regionales: Proviene del volcán Miravalles.

Relaciones estratigráficas: Cubre a los flujos pumíticos. En una perforación geotérmica cerca de Fortuna se alcanzó al menos un espesor de $100 \mathrm{~m}$.

Edad geológica: Las edades de radiocarbono nos suministran edades de 8300 años atrás, 
correspondientes al Holoceno (Alvarado et al., 2004).

Aspectos específicos: Se trata de un debris avalanche diluido a debris flow.

Antecedentes: Por muchos años se le asocio con un lahar hasta que con los estudios de detalle y los nuevos conocimientos vulcanológicos, se esclareció su origen (ver Alvarado et al., 2004 para los detalles históricos).

\section{Depósitos epiclásticos}

Descripción: Está constituido por sedimentos fluviales (desde cantos aluviales muy gruesos hasta arenas y limos como sedimentos finos de inundación) transportados por los ríos Cuipilapa y Tenorio y por la quebrada Santa Fé y sus tributarios. Los constituyentes son lavas y arenas vidriosas o pumíceas.

Localidades: se encuentran principalmente en el sector centro y centro-sur de la hoja.

Aspectos regionales: Forma parte de las porciones más elevadas de las llanuras entre el río Tenorio y Casa Vieja del Norte.

Relaciones estratigráficas: Sobreyace a la mayoría de las unidades precedentes.

Edad geológica: No hay edades absolutas, pero debe de quedar comprendido entre el Pleistoceno Superior y el Holoceno, con excepción de aquellos que infrayacen a las lavas de Monteverde, siendo estos últimos de edad Pleistocena Inferior.

\section{ESTRUCTURAS}

Los estudios estructurales se han restringido en su mayoría a estudios de alineamientos y algunos afloramientos. En el área estudiada se encuentran estructuras antiguas de rumbo NE-SW, difícilmente distinguible en el campo y en fotografías aéreas. Además, se encuentran otras alineaciones mas recientes de rumbo N-S, que están colegadas a fallas sinestrales. Estas estructuras tienen un desarrollo longitudinal generalmente limitado, excepto por el graben de La Fortuna que es muy bien definido por escarpes morfológicos rectilíneas y que interfiere en particular con el desarrollo del retículo hidrográfico (véase el desarrollo rectilíneo de los ríos Aguacaliente y Blanco). Las mejores evidencias tectónicas se encuentran en las capas piroclásticas de la Meseta de Santa Rosa: en todos los flujos piroclásticos se encuentran fracturas y fallas con rumbo N-S, secundariamente con rumbo NE-SW y E-W. Las fallas son generalmente verticales, aunque solo en pocos casos se ha reconocido en el campo la dirección de movimiento. Dos eventos sísmicos importantes se presentaron en la primera mitad del siglo XX en los alrededores de Bagaces (Montero \& Alvarado, 1988).

\section{AGRADECIMIENTOS}

Al Instituto Costarricense de Electricidad el Consejo Nacional de Investicaciones (CNR) y a la Universidad de Milán por todo el apoyo brindado en el campo y en el laboratorio. A Benjamín Acevedo por su labor en la edición final del mapa. Estos últimos trabajos han sido el fruto de un programa de colaboración entre el ICE (Instituto Costarricense de Electricidad), el CNR (Consiglio Nazionale delle Ricerche, Italia) y el Dipartimento di Scienze delle Terra den' Università di Milano, Italia.

\section{REFERENCIAS}

AIAZZI, D., FIORLETTA, M., CIVELLI, G., CHIESA, S. \& ALVARADO, G.E., 2004. Geología de la Hoja Cañas. - Rev. Geol. Amér. Central, 30: 215-223.

ALVARADO, G.E., 2000: Volcanes de Costa Rica: Geología, historia y riqueza natural. - 269 págs. EUNED, San José. 
ALVARADO, G.E., KUSSMAUL, S., CHIESA, S., GILLOT, P.-Y, WÖRNER, G. \& RUNDLE, C, 1992: Cuadro cronoestratigráfico de las rocas ígneas de Costa Rica basado en dataciones radiométricas. - J. South Amer. Earth Sci. 6(3): 151-168.

ALVARADO, G.E., VEGA, E., CHAVES, J. \& VÁZQUEZ, M., 2004. Los grandes deslizamientos (volcánicos y no volcánicos) de tipo debris avalanche en Costa Rica. - Rev. Geol. Amér. Central, 30: 83-99.

CHAVES, R. \& SÁENZ, R., 1974: Geología de la cordillera de Tilarán (Proyecto Aguacate, 2a fase). - Dirección Geol. Minas y Petról., San José, Inf. Téc. Notas Geol. 12(53): 11-49.

CHIESA, S., 1991: El flujo de pómez biotítica del Río Liberia (Guanacaste), Costa Rica, América Central. - Rev. Geol. Amér. Central., 12: 73-83.

CHIESA, S., CORELlA, M. \& MORA, O., 1987: Geología de la Meseta ignimbrítica de Santa Rosa, Guanacaste, Costa Rica. -133 págs., Instituto Costarricense de Electricidad [Informe interno].

CHIESA, S., CIVELli, G., GYLlOT, P.-Y., MORA, O. \& ALVARADO, G.E., 1992: Rocas piroclásticas asociadas con la formación-de la Caldera de Guayabo, Cordillera de Guanacaste, Costa Rica. Rev. Geol. Amér. Central, 14: 59-75.

CHIESA, S., ALVARADO, GE., PECCHIO, M., CORELLA, M. \& ZANCHI, A., 1994: Contribution to petrological and stratigraphical understanding of the Cordillera de Guanacaste lava flows, Costa Rica. - Rev. Geol. Amér. Central, 17: 19-43.

CIGOLINI, C. \& CHAVES, R., 1986: Geological, petrological and metallogenic characteristics of the Costa Rica gold belt: contribution to new explorations.

- Geol. Rundsch. 75(3): 737-754.

CIVELLI G. 1990: Geovulcanologia e petrochimica della Cordigliera del Guanacaste (Costa Rica): le piroclastiti dell'area Chorotega. - 165 págs. Univ. Milano [Tesis de Laurea].

DENGO, G., 1962: Estudio geológico de la región de Guanacaste, Costa Rica. 112 págs.; Instituto Geográfico de Costa Rica, San José.

GILLOT, P.-Y, CHIESA, S. \& ALVARADO, GE., 1994: Chronostratigraphy of Upper Miocene-Quaternary volcanism in northern Costa Rica. - Rev. Geol. Amér. Central, 17: 45-53.

ICE-ENEL, 1988: Estudio de reconocimiento y prefactibili-dad geotérmica en la República de Costa Rica, Fase I. - 337 págs. Ente Nazionale per l'Energia Eléctrica - ICE [Informe interno].

LOCATI, U., 1990: Geología del versante meridionale del Volcan Tenorio, Costa Rica. - 85 págs. Univ. Milano [Tesis de Laurea].

MONTERO, W. \& ALVARADO, G.E., 1988: Los terremotos de Bagaces de 1935 y 1941: Neotectonismo transversal a la Cordillera volcánica del Guanacaste, Costa Rica. - Ciencia y Tecnología, 12 (1-2): 69-87.

MORA, O., 1988: Estudio geológico-petrológico de las piroclastitas al los alrededores de Bagaces, provincia de Guanacaste, Costa Rica. - 61 págs.; Escuela Centroamericana de Geología, Univ. de Costa Rica [Tesis Lic.].

PECCHIO, M., 1990; Geovolcanologia e petrochimica della Cordigliera di Guanacaste (Costa Rica): le lave dell’ area Chorotega. 
-189 págs. Tesis de Laurea, Univ. degli Studi di Milano, Dipartimento di Scienze della Terra.

RAMÍREZ, O., 1973: Estudio geológico y geotécnico de una faja de terreno atravesado por el posible trazado de la carretera entre Tilarán y Fortuna. - 87 págs, Escuela
Centroamericana de Geología, Univ. Costa Rica [Tesis Lic.].

VOGEL, T.A., PATINO, L.C., ALVARADO, G.E. \& GANS. P.B., 2004. Silicic ignimbrites within the Costa Rican volcanic front: evidence for the formation of continental crust. - Earth Planet. Sci. Lett. 226: 149-159. 


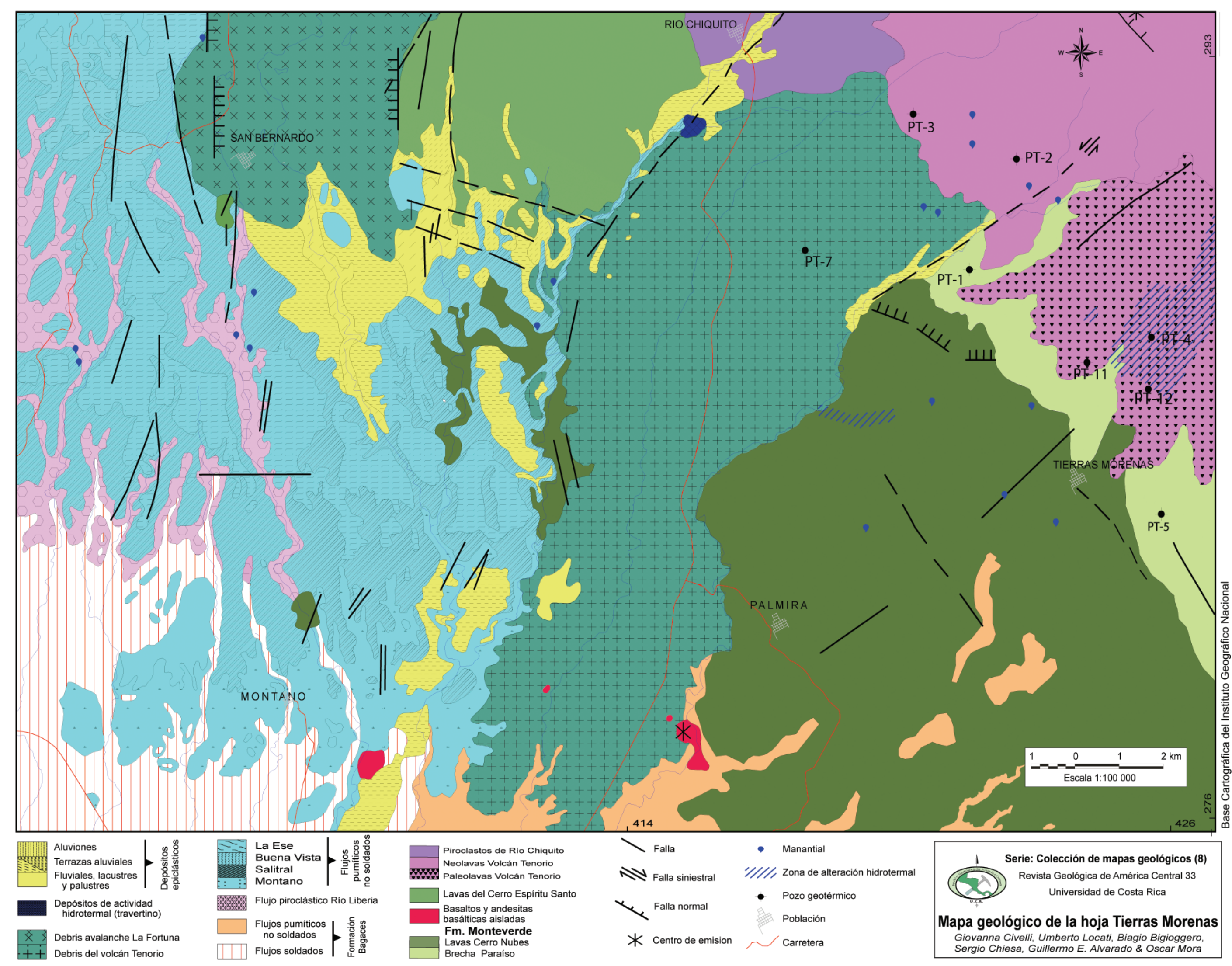

